\title{
TITLE: PREDICTING MASSIVE BLOOD LOSS IN CARDIAC SURGERY
}

AUTHORS: Rachel O'Farrell MD, Keyvan Karkouti MD - for the Reducing Bleeding in Cardiac surgery (RBC) Research Group.

AFFILIATIONS: Department of Anesthesia \& Pain Management, Toronto General Hospital, University Health Network, University of Toronto, 3ES-402, 200 Elizabeth Street, Toronto, ON, Canada M5G 2C4

INTRODUCTION: Massive blood loss (MBL) is a common and serious complication of cardiac surgery. It occurs in $10 \%$ of patients and is associated with increased morbidity and mortality. Given the heavy burden of illness of MBL in cardiac surgery, an accurate clinical prediction rule for identifying at-risk patients would be valuable. In this study, we developed and internally validated such a prediction rule.

METHODS: Following institutional approval, data on all patients having undergone cardiac surgery with CPB from 1999 to 2004 at a single tertiary care hospital were obtained from validated clinical databases. MBL, the dependent variable, was defined as the transfusion of more than five units of RBC. The independent variables were perioperative variables associated with perioperative blood loss. Multivariable logistic regression analysis was used to develop the prediction rule on the first $80 \%$ of the sample population. An appropriate probability cut-off, for classifying patients at high-risk for MBL, was identified. The prediction rule was then validated on the remaining $20 \%$ of the sample.

RESULTS: Complete data were available on 10,132 patients. The logistic regression model, developed on the first 8,102 patients, includes 11 variables, with the 4 most predictive variables being CPB duration, baseline hemoglobin, urgency of procedure, and renal function. The model is well calibrated (Hosmer-Lemeshow test $\mathrm{P}=0.64$ ) and is highly discriminative when tested on the patients in the training set (ROC area 0.83). Prospective validation showed that the prediction rule at the probability cut-off of 0.2 has a positive predictive value of $55 \%$ and negative predictive value of $89 \%$ of detecting patients transfused at least five units of RBC.

DISCUSSION: Using this prediction rule, one can accurately identify which patients are likely to develop MBL after surgery. This information can be used reduce this risk by modifying clinical care. As well, the rule can be used to efficiently research the safety and effectiveness of novel therapies for MBL by allowing the targeting of only high-risk patients for recruitment in clinical studies. To this end, we are currently in the process of assessing the external validity of this prediction rule at six Canadian hospitals.

ACKNOWLEDGEMENT: The Canadian Institutes of Health Research, Canadian Blood Services, and Novo Nordisk provided Support for this work. 